Anna Volodina

\title{
Null ist nicht gleich Null: \\ Zur diachronen Entwicklung von Nullsubjekten im Deutschen
}

\section{Einleitung ${ }^{1}$}

Der Aufsatz beschäftigt sich mit Nullsubjekten im Deutschen, einem Phänomen, das aus diachroner Sicht in der Forschung bisher stark vernachlässigt wurde. Der Grund dafür ist die (immer noch) verbreitete Meinung, dass ahd. Nullsubjekte - bedingt durch die sklavische Wortfür-Wort-Übersetzung aus dem Lateinischen - nicht als Teil der nativen ahd. Grammatik angesehen werden (vgl. Schrodt 2004: 73f.). Allerdings lässt sich nach neueren empirisch basierten Arbeiten zu diesem Thema die Lehnsyntax-Hypothese für das AHD nicht länger halten (vgl. Fleischer 2006, Axel 2007, Schlachter 2010). In diesem Aufsatz wird aufgrund empirischer Evidenz für eine sprachgeschichtliche Kontinuität des Nullsubjekt-Phänomens argumentiert, da ähnliche Kontexte und die Lizenzierungsbedingungen, unter denen Nullsubjekte im FNHD und frühen NHD vorkamen, Überschneidungen einerseits mit dem AHD, andererseits mit den heutigen Dialekten offenbaren, wobei die Variationsbreite von Nullsubjekten in den früheren Sprachstufen viel größer ist als heute.

Der Aufsatz ist wie folgt aufgebaut: Zunächst diskutiere ich, wie sich das heutige Deutsch im Vergleich zu pro-drop-Sprachen verhält (Abschnitt 2). Danach zeige ich anhand ahd. Daten und neuerer Untersuchungen zum FNHD und frühen NHD die diachrone Entwicklung der Nullsubjekt-Eigenschaft (Abschnitt 3). Überlegungen zur Notwendigkeit einer weiteren systematischeren Erforschung der Diachronie von Nullsubjekten bilden den Abschluss (Abschnitt 4).

1 An dieser Stelle bedanke ich mich bei allen Diskutanten für ihr Feedback zum Vortrag. Für hilfreiche Kommentare und Hinweise zu einer früheren Fassung dieses Aufsatzes danke ich Thomas Strobel und Helmut Weiß. Bei Maria Theresa Distler bedanke ich mich für die Unterstützung bei der Annotation von FNHD-Daten. 


\section{Deutsch und Nullsubjekt-Sprachen}

Wie die meisten Sprachen der Welt zählen viele romanische und westslawische Sprachen zu den Sprachen, die pronominale Subjekte nicht notwendigerweise explizit realisieren müssen, nicht aber die germanischen, inklusive des heutigen Standarddeutschen. Die Auslassung des Subjektpronomens im Mittelfeld eines Hauptsatzes wie in (1), in einem eingebetteten Satz wie in (2) oder W-Fragesatz wie in (3) sowie die Auslassung eines semantisch leeren Expletivums wie in (4) ist im Deutschen ungrammatisch.

(1) a. Wahrscheinlich arbeitet *(er) in Heidelberg.

DEU

b. Probabilmente $[\varnothing]$ lavora a Heidelberg.

ITA

(2) a. Peter ${ }_{\mathrm{j}}$ sagte $\mid$ dass * $\left(\mathrm{er}_{\mathrm{j}}\right)$ unwiderstehlich ist.

DEU

b. Ha detto $\mid$ che $[\varnothing]$ è irresistibile.

ITA

(3) a. Wann kommt*(er)?

DEU

b. Quando viene $[\varnothing]$ ?

ITA

(4) a. *(Es) regnet.

b. [Ø] Piove.

DEU

ITA

Dagegen ist die Setzung eines Subjektpronomens im Italienischen unter denselben syntaktischen Bedingungen keinesfalls obligatorisch, daher bleibt es im nicht-markierten Fall phonetisch leer. ${ }^{2}$ Im Rahmen der generativen Grammatik geht man davon aus, dass die Subjektpronomina in (1b), (2b), (3b) oder (4b) nur auf der Oberfläche weggelassen werden, d.h., in der syntaktischen Repräsentation sind sie als leeres Pronomen (pro) vorhanden. Hinsichtlich dieser Eigenschaft wird das Italienische den pro-drop-Sprachen (engl. pro-drop languages), das Deutsche im Gegenteil den Nicht-pro-drop-Sprachen zugeordnet. Dieser Kontrast wird in der Regel (vgl. Rizzi 1986) auf morphologische Eigenschaften der jeweiligen Sprachen zurückgeführt: Anders als Nicht-pro-drop-Sprachen wie Deutsch und Englisch sind pro-drop-Sprachen wie Italienisch Sprachen mit reicher Flexion am Verb, die eine eindeutige Markierung der Kongruenzkategorien erlauben, was die leere Kategorie pro identifiziert und lizenziert. Umgekehrt muss das Englische und auch das Deutsche das Pronomen durch lexikalische Realisierung anzeigen.

$\begin{array}{llll} & \text { ITA [+pro-drop] } & \text { ENG [-pro-drop] } & \begin{array}{l}\text { DEU [-pro-drop] } \\ \text { sprech - e }\end{array} \\ \text { 1.SG } & \text { parl - o } & \text { speak } & \text { sprich }- \text { st } \\ \text { 2.SG } & \text { parl - i } & \text { speak } & \text { sprich }- \text { t } \\ \text { 3.SG } & \text { parl - a } & \text { speak }-\mathrm{s} & \text { sprech }- \text { en }\end{array}$

2 Die Stelle des leeren Pronomens wird durch $\varnothing$ in diesen und weiteren Beispielen verdeutlicht. 
2.PL parl - ate

3.PL parl - ano speak

speak sprech- $\mathrm{t}$

sprech - en

Das Verbparadigma des Italienischen - einer prototypischen pro-dropSprache - unterscheidet sechs verschiedene Formen, das des Englischen dagegen nur zwei, so dass in der Tat keine Eindeutigkeit der Personenmarkierung am Finitum vorhanden ist. Das Deutsche mit fünf - wenn man auch die innere Flexion berücksichtigt - verschiedenen Formen für den Indikativ eines starken Verbs wie sprechen ist zwar nicht so eindeutig wie das Italienische, aber nur bedingt als „arm“ zu bezeichnen. ${ }^{3}$ Gegen die Annahme einer Eins-zu-eins-Beziehung zwischen reicher Flexion und pro spricht die Tatsache, dass es sowohl pro-drop-Sprachen gibt, die morphologisch hinsichtlich Person-Numerus-Kongruenz nicht markiert sind wie das Japanische, das keine Person-Numerus-Flexion kennt (vgl. Huang 1989, Jaeggli/Safir 1989), als auch Nicht-pro-drop-Sprachen mit reicher Flexion wie das Isländische: Trotz reicher Flexionsmorphologie sind Nullsubjekte im Isländischen auf semantisch nicht gehaltvolle Subjekte (Expletiva) beschränkt (vgl. (5); Haider 2001: 286), in anderen Fällen muss das Subjektpronomen overt realisiert werden:
(5) í gœr rigndi.
ISL
gestern regnete-3.SG
„Gestern regnete es."

Dass Expletiva einen Sonderfall darstellen, lässt sich auch am Beispiel des Deutschen zeigen. Selbst in der Nicht-pro-drop-Sprache Deutsch gibt es eine Position im Mittelfeld, in der Nullexpletiva bei unpersönlichen Passiven obligatorisch sind (vgl. Grewendorf 1989), wie in (6b) und (6c) gezeigt:

(6) a. Es wurde auf der Hochzeit viel getanzt.

b. Auf der Hochzeit wurde *es/[Ø] viel getanzt.

c. Der Mann erzählte, dass *es/[Ø] auf der Hochzeit getanzt wurde.

Werden standardsprachliche Strukturen wie in (6a), wo es als Platzhalter im Vorfeld eines Hauptsatzes realisiert werden muss, im gesprochenen Deutsch oder in den deutschen Dialekten geäußert, ist das Subjektpronomen optional zu setzen, vgl. (6a'):

(6) a'. Wie ist die Hochzeit gelaufen? (Es) Wurde viel getanzt.

3 Gereon Müller (2006) sieht systematische Synkretismen im Paradigma der Verbalflexion als Blockierer für pro. Die Synkretismen betreffen die 1 und 3SG.Prät., die 1 und 3PL, die 3SG und 2PL.Präs. sowie 2 und 3SG.Präs., die das Flexiv/t/ gemeinsam haben. 
Auch referentielle Subjekte (7a und b) und - wenn auch seltener - Objekte (7c), sofern sie aus dem Ko- oder Kontext rekonstruierbar sind, können unter bestimmten pragmatischen Bedingungen (z. B. in Frage-AntwortSequenzen) im Vorfeld selbständiger V2-Sätze getilgt werden: ${ }^{4}$

(7) a. Wo war denn Peter ${ }_{j}$ gestern? [Ø] $]_{j}$ Hat gestern zu Hause gearbeitet.

a.' Wo war denn Peter gestern? Gestern hat *(er) zu Hause gearbeitet.

b. Morgen ist die Institutsparty! [Ø] Bin auch eingeladen!

b.' ICH bin auch eingeladen, DU nicht!

c. Gehst du mit ins Kino? [Der neue Harry Potter $]_{\mathrm{j}}$ läuft. [Ø] $]_{\mathrm{j}}$ Habe ich schon gesehen.

c.' Gehst du mit ins Kino? Der neue Harry Potter läuft. Ich habe *(ihn) schon gesehen.

Anders als in den pro-drop-Sprachen (und auch im schriftlichen Standarddeutschen) steht den Nullargumenten im gesprochenen Deutsch 5 ausschließlich die sogenannte Topik-Position zur Verfügung. Wird das Vorfeld des Satzes anderweitig besetzt, muss das Subjekt- bzw. Objektpronomen postfinit in der Wackernagelposition obligatorisch gesetzt werden (vgl. 7a' und 7c'). Das erklärt auch, warum Konstruktionen, in denen Topik-drop im Gegenwartsdeutschen vorkommen kann, ausschließlich Matrix-Sätze sind: In $w$-Sätzen (Interrogativ-, Exklamativsätze, Entscheidungsfragen) und Nebensätzen (inklusive eingebettete V2-Sätze) muss das Subjektpronomen dagegen overt realisiert werden (vgl. 2a und 3a). Neben dieser syntaktischen Beschränkung wird eine wichtige Rolle der Informationsstruktur beigemessen: Topikale Elemente können nur dann weggelassen werden, wenn sie keinen Kontrastfokus haben wie in $\left(7 b^{\prime}\right)$ oder wenn kein Topikwechsel vorliegt. Sie müssen außerdem koindiziert auftreten, d.h., nur Elemente mit derselben syntaktischen Funktion können getilgt werden (vgl. 7a und 7c); (für weitere Bedingungen für Topik-drop siehe Fries 1988). Für Topik-drop gibt es keine morphologischen Beschränkungen. D. h., in der Topik-Position können Subjektpronomina aller Personen und Numeri weggelassen werden, unabhängig davon, ob das Antezedens in der 3.SG/PL belebt oder unbelebt auftritt. In der 1.SG/PL referieren Nullsubjekte ausschließlich auf Personen, sie werden ohne anaphorischen Bezug meist in bestimmten (konzeptionell mündlichen) Textsorten wie Privatbrief, E-Mail, Chat oder Tagebuch verwendet, daher werden sie in der Literatur unter Diary-drop (siehe Haegeman 2000 für das Englische) geführt.

4 Unter (7) sind keine Koordinationsellipsen aufgeführt.

5 Das Deutsche soll hier nicht als ein grammatisch monolithes System verstanden werden. 
Schon anhand dieser wenigen Beispiele kann man mit Sicherheit zeigen, dass das gesprochene Deutsch (im Kontrast zum konzeptionell schriftlichen Standarddeutsch) in gewissem Sinne eine Nullsubjekt-Sprache ist, die einer eigenständigen Behandlung bedarf. Die oben aufgelisteten Lizenzierungsbedingungen für Nullsubjekte im (gesprochenen) Deutsch unterscheiden sich aber systematisch in entscheidenden Punkten von den Lizenzierungsbedingungen für pro im Italienischen. Diese lassen sich wie folgt tabellarisch festhalten:

\begin{tabular}{|l|l|l|}
\hline & Gespr. DEU & ITA \\
\hline Möglichkeit für (referentiellen) Subjekt-drop & $\sqrt{ }$ & $\sqrt{ }$ \\
\hline - diskursabhängig & $\sqrt{ }$ & - \\
\hline - positionsabhängig & $\sqrt{ }$ & - \\
\hline Möglichkeit für (nicht-referentiellen) Expletiv-drop & sehr eingeschränkt & $\sqrt{ }$ \\
\hline Möglichkeit für Objekt-drop & $\sqrt{ }$ & - \\
\hline Sprachtyp & Topik-drop & pro-drop \\
\hline
\end{tabular}

Tab 1: Unterschiede zwischen dem gespr. DEU und ITA bzgl. Nullsubjekt-Eigenschaft

Im Unterschied zur pro-drop-Sprache Italienisch wird (gesprochenes) Deutsch in der Forschung generell als Topik-drop-Sprache behandelt (spätestens seit Huang 1984). Abgesehen von Fällen mit obligatorischen Nullexpletiva bei unpersönlichem Passiv sprechen die strukturellen Eigenschaften des Deutschen gegen die Annahme von pro-drop. Das gesprochene Deutsch ist und war eine Topik-drop-Sprache - das ist unumstritten. Die Frage, ob sich das ältere Deutsch gegebenenfalls auf die Topik-dropEigenschaft reduzieren lässt, oder diachron doch mehr Gemeinsamkeiten als Unterschiede zu prototypischen pro-drop-Sprachen aufweist als bisher angenommen, soll im nächsten Kapitel erörtert werden.

\section{Nullsubjekt-Eigenschaft und Diachronie des Deutschen}

\subsection{Nullsubjekte im Althochdeutschen}

In der Forschung wird nach wie vor kontrovers diskutiert, ob das AHD und die altgermanischen Sprachen im Allgemeinen (referentielles) prodrop aufwiesen oder nicht. Abgesehen von der theoretischen Debatte über die Art von ahd. „Subjektlücken“, die hier nicht weiter verfolgt wird, stellt die überlieferte Beleglage große Schwierigkeiten für die Forschung dar. Die frühesten Texte des AHD weisen eine relativ hohe Anzahl von 
Nullsubjekten auf, während sie bei Notker kaum belegt sind, ${ }^{6}$ was intuitiv den Schluss erlaubt, dass in der ahd. Grammatik Nullsubjekte nicht vorgesehen waren. Auf den ersten Blick scheint die empirische Beobachtung, die Eggenberger (1961) macht, eine Erklärung dafür zu liefern, warum die Nullsubjekt-Eigenschaft nicht als natives Phänomen der ahd. Grammatik angesehen werden kann: „Es sind nicht die ältesten Texte, die das Subjektpronomen vermissen, sondern die lateinischsten" (Eggenberger 1961: 167). Bei den ältesten überlieferten Texten (z. B. Isidor, Tatian) handle es sich mit hoher Wahrscheinlichkeit um Wort-für-Wort-Übersetzungen aus dem Lateinischen, das bekanntlich eine pro-drop-Sprache ist. Daher sei das hohe Vorkommen von Nullsubjekten gerade in diesen Texten nicht überraschend. Während die älteren Arbeiten an der Lehnsyntaxhypothese festhalten, lassen die neueren Untersuchungen zum AHD von Fleischer (2006), Axel (2007) und zuletzt Schlachter (2010), die sich nicht nur auf Eggenbergers Auszählungen beruft, sondern auch eigene Erhebungen in die Analyse einbezieht, keinen Zweifel daran, dass die Nullsubjekt-Eigenschaft ein natives Phänomen des AHD ist: Mit der Lehnsyntaxhypothese lässt sich nicht erklären, warum die Distribution von Nullsubjekten im AHD anders als im Lateinischen ist, insbesondere

- warum Subjektpronomina gegen die lateinische Vorlage gesetzt werden (vgl. Belege aus Tatian in Fleischer/Hinterhölzl/Solf 2008: 225),

- warum Nullsubjekte nur in bestimmten grammatischen Strukturen lizenziert sind (Axel 2007) und schließlich

- warum im Isidor der Zitatteil, der in besonderer Weise unter dem Einfluss des Lateinischen steht, nicht mehr, sondern weniger Nullsubjekte aufweist als der Traktatteil (siehe eine neue Evaluierung ahd. Daten in Schlachter 2010).

Axel (2007) argumentiert gegen die Lehnsyntaxhypothese und für die Annahme von pro-drop im AHD. Sie zeigt, dass vor allem bei den ältesten ahd. Texten (Isidor, Monseer Fragmente und Tatian) eine asymmetrische Distribution in Bezug auf die Person bei Subjektauslassungen besteht. Pronominale Subjekte werden auffällig häufiger in der 3.SG und PL ausgelassen: „Referential null subjects are attested in all persons and nambers. However [...], it is only in third person singular and plural that the null variant is used more frequently than the overt one" (Axel 2007: 314). Eine solche personenspezifische Verteilung zwischen overt und covert realisier-

6 Nach der Auszählung von Eggenberger (1961) wird bei Isidor (vor 800) etwas weniger als ein Drittel (28\%) aller Subjektpronomina overt realisiert, bei Tatian (vor 850) ca. 40\%, dagegen finden sich bei Notker (10. Jh.) und Williram (11. Jh.) jeweils nur noch 4 Belege für Nullsubjekte. 
ten Subjektpronomina ist für die pro-drop-Sprache Latein untypisch, weil pro-drop-Sprachen generell keine morphologische Restriktion haben. Die Verbalmorphologie allein (mit einer Ausnahme in der 1.PL) kann aber das Vorkommen von Nullsubjekten im AHD nicht lizenzieren, stattdessen bestehe nach Axel (2007) ein direkter Zusammenhang zwischen der Art der Verbstellung und der Möglichkeit, Subjekte auszulassen: Tendenziell begünstigen V2-Sätze Nullsubjekte, in den VE-Sätzen muss dagegen das Subjektpronomen overt realisiert werden. Eine solche asymmetrische syntaktische Distribution ist für das Lateinische wiederum untypisch. Die Generalisierung lautet: Nullsubjekte sind nur dann lizenziert, wenn sie postfinit realisiert werden (Axel 2007: 313) wie in (8):

(8) Sume hahet $[\varnothing]$ in cruci einige hängt (ihr) in kreuz (MF XVIII,17; AHD, "Einige werdet ihr kreuzigen." zit. nach Axel 2007: 293)

Dies würde erklären, warum ahd. Nullsubjekte in VE-Sätzen overt realisiert werden. Die Möglichkeit zur postfiniten Realisierung von Nullsubjekten ist im heutigen Standarddeutschen nicht gegeben, dafür lassen sich solche Nullsubjektrealisierungen analog zu Nullsubjekten in der prodrop-Sprache Italienisch analysieren, was Axel dazu veranlasst, pro-drop im AHD anzunehmen, das - anders als in den prototypischen pro-dropSprachen - syntaktisch lizenziert wird. Schlachter (2010) verweist außerdem darauf, dass neben post- auch präverbale Nullsubjekte nicht selten belegt seien:

(9) „Sus quhad druhtin uuerodheoda got: $[\varnothing]$ sendida mih after guotliihin zi dheodom,

So sprach (der) Herr, der Heer Gott: (er) sandte mich zu seinem Ruhm zu den Völkern

,Hęc dicit dominus deus exercituum: Post gloriam misit me ad gentes (Is. III.8, Eg. 217-219, He 11,17-18, zit. nach Schlachter 2010)

Daten wie (9) würden zwar der von Axel (2007) vorgeschlagenen Analyse, dass pro durch die Präsenz von Flexion in $\mathrm{C}^{0}$ /in der linken Satzklammer lizenziert wird, widersprechen, der Idee einer partiellen pro-drop-Sprache AHD stünden sie trotzdem nicht im Wege. Außerdem ist gegen Schlachter (2010) zu erwähnen, dass es im AHD V1-Deklarative gab, so dass eine postfinite Ansetzung von pro bei Fällen wie (9) nicht auszuschließen ist (vgl. Axel 2007: 311f.). 


\subsection{Nullsubjekte im Übergang zum NHD}

Wenn man das AHD mit Axel (2007) als partielle pro-drop-Sprache analysiert, stellen sich die Fragen, ob und ab wann (referentielles) pro-drop in der Geschichte des Deutschen nicht mehr möglich ist und mit welchen grammatischen Restriktionen dies zusammenhängen kann. Die Antworten auf diese Fragen hängen miteinander zusammen.

\subsubsection{Verlust oder Etablierung von Nullsubjekten?}

Die Standardmeinung besagt, dass Nullsubjekte im Übergang zum MHD verschwinden. Als Ursache für den Verlust von Nullsubjekten wurde die Verarmung der Flexionsparadigmen im Übergang vom AHD zum MHD diskutiert, wodurch morphologische Transparenz nicht mehr gewährleistet werden konnte und Subjektpronomina obligatorisch wurden. Dass Flexionsmorphologie nicht zwangsläufig in einem direkten kausalen $\mathrm{Zu}$ sammenhang mit der Nullsubjekt-Eigenschaft steht, wurde bereits in Abschnitt 2 diskutiert. Demnach kann der Abbau der Flexionsmorphologie nicht der (einzige) Grund für den Verlust von Nullsubjekten in den früheren Sprachstufen des Deutschen sein. ${ }^{7}$ Außerdem kann mit flexionsmorphologischem Wandel nicht erklärt werden, warum der Rückgang von Nullsubjekten nicht wie bisher angenommen im Übergang zum MHD, sondern schon viel früher stattfindet, und zwar im Übergang vom mittleren zum späteren $\mathrm{AHD},{ }^{8}$ wo keine nennenswerten diachronen Veränderungen morphologischer und syntaktischer Art aufgetreten sind. Welche Faktoren einen Nullsubjektverlust tatsächlich ausgelöst haben, darüber kann nach wie vor wegen der spärlichen Datenlage nur spekuliert werden.

Dass in den mhd. Verstexten, die meistens als Grundlage für die Untersuchungen des MHD dienen, das Subjektpronomen stets overt realisiert wird, ist lange noch kein Grund für die Annahme, dass Nullsubjekte, welcher Art auch immer sie sind, endgültig verschwunden sind. Diese Veränderung kann auch der Textsorte bzw. dem durch die Textsorte variierenden Sprachgebrauch geschuldet sein. Offensichtlich muss auch bei der Analyse historischer Texte das Spannungsverhältnis zwischen der stan-

7 Als mögliche Ursachen dafür werden in der Literatur außerdem syntaktischer Wandel mit der Entwicklung vom synthetischen zum analytischen Satzbau (Nübling et al. 2006) und die Obligatorisierung des Subjektpronomens als eine Folge dessen Grammatikalisierung (Szczepaniak 2009) diskutiert.

8 Bei Notker (10. Jh.) bzw. Williram (11. Jh.) sind Nullsubjekte kaum belegt (siehe auch die Fußnote 6). 
dardisierten Form des Deutschen, der dialektal geprägten Syntax und der (konzeptionell) mündlich geprägten Syntax (dazu zuletzt Fleischer 2010) mitberücksichtigt werden. Eine Differenzierung zwischen Dialektsyntax, Syntax des (konzeptionell) gesprochenen und Syntax des (konzeptionell) geschriebenen Standards ist generell dann sinnvoll, wenn ein bestimmtes grammatisches Phänomen niemals oder äußerst selten in den gleichen Kontexten in den drei genannten Varietäten des Deutschen anzutreffen ist. Für die Realisierung von Nullsubjekten würde das heißen, dass das Nullsubjekt-Phänomen die grammatischen Regeln einer Varietät befolgt, aber nicht zwangsläufig der anderen. ${ }^{9}$ Dieses Argument scheint auf einer soliden empirischen Evidenz zu beruhen. Anknüpfungspunkte ergeben sich zur Situation in den meisten heutigen Dialekten des Deutschen, wie hier am Beispiel des Bairischen dargestellt, vgl. (10). Wie im AHD können auch in den Dialekten Nullsubjekte im Mittelfeld eines V2-Satzes optional realisiert werden, vgl. (10a). Ist die subordinierende Konjunktion dagegen wie das Verb flektiert, kann das pronominale Subjekt in derselben strukturellen Konstellation zu den Kongruenzmerkmalen wie in (10a) stehen und darf daher auch in einem VE-Satz covert realisiert werden, wie in (10b) gezeigt.

(10) a. I glaub, morng sads [Ø] wieder gsund.

b. Wennsd [Ø] af Minga kimsd. (zit. nach Weiß 1998: 125)

Obwohl Sätze wie (10b) im AHD nicht belegt sind, zeigen sie eine klare Parallele zu den in Abschnitt 3.1 beschriebenen ahd. pro-drop-Strukturen. ${ }^{10}$ Nullsubjekte wie in (10b) unterliegen denselben Lizenzierungsbedingungen wie pro-drop im AHD: Sie werden im Mittelfeld nach der Flexion in $\mathrm{C}^{0}$ /in der linken Satzklammer realisiert. Der Analyse in Axel/Weiß (2010) folgend lassen sich einerseits Erweiterung der pro-drop-Eigenschaft auf Nebensätze, andererseits aber auch morphologische Einschränkungen in Bezug auf die Person feststellen, da sie nur bei pronominaler Flexion, d. h. hauptsächlich in der 2.SG/PL lizenziert sind. Die relevante Entwicklung in der Lizenzierungsbedingung fand wahrscheinlich im späten AHD statt. Identische Lizenzierungsbedingungen für pro im AHD und in der heutigen Dialektsyntax sprechen möglicherweise für eine kontinuierliche Entwicklung der Nullsubjekteigenschaft im Deutschen und dafür, dass

9 Ähnlich argumentieren auch Kaiser/Meisel (1991) für das Französische, das „,in einigen Varietäten zutreffend als Null-Subjekt-Sprache beschrieben werden kann, während andere Varietäten nicht die entsprechenden syntaktischen Charakteristika aufweisen" (ebd., 110).

10 Nullsubjekte wie in (10) werden in Bayer (1984: 378) und Weiß (1998: 116ff., 2005) als pro-drop interpretiert. 
solche Strukturmuster mit hoher Wahrscheinlichkeit in mhd. und fnhd. Texten der entsprechenden Varietät zu finden sind (vgl. Axel/Weiß 2010).

\subsubsection{Nullsubjekte im FNHD und im frühen NHD}

Um die Veränderungen bei der diachronen Entwicklung von Nullsubjekten im Deutschen nachvollziehen zu können, ist eine detaillierte Analyse aller historischen Sprachstufen des Deutschen nötig, allerdings fehlen bislang verlässliche Daten für das MHD und FNHD. Die einzige umfassende empirische Arbeit, die sich mit referentiellen Nullsubjektstrukturen im MHD und FNHD beschäftigt, ist immer noch die von Held (1903). Diese genügt aber heute weder den methodischen noch theoretischen Ansprüchen. Am Lehrstuhl für Historische Sprachwissenschaft (Institut für Linguistik) an der Goethe-Universität Frankfurt wird eine neue, empirisch breit angelegte Korpusstudie zu Nullsubjekten im MHD und FNHD vorbereitet.

Schon im Vorfeld dieser größeren Studie wurden im Rahmen kleinerer korpusbasierter Pilotstudien zum frühen NHD und FNHD die in Axel (2007) und Axel/Weiß (2010) aufgestellten Hypothesen ansatzweise empirisch überprüft. Eine Analyse zweier nichtliterarischer Texte um $1650^{11}$ (liegt mit Volodina 2009 publiziert vor) und eines frühneuhochdeutschen Texts um $1450^{12}$ zeigen, dass referentielle und nicht-referentielle Nullsubjekte der erwähnten Zeitabschnitte einerseits erstaunliche Parallelen zum AHD, andererseits aber auch deutliche syntaktisch-strukturelle, referentielle sowie paradigmatische Unterschiede zur Situation im heutigen Deutsch aufweisen. $\mathrm{Ob}$ und welche Unterschiede sich zwischen dem MHD und FNHD im Allgemeinen und zwischen den einzelnen Varietäten der untersuchten Sprachstufen in Bezug auf die Distribution und Typologie von Nullsubjekt-Strukturen feststellen lassen, soll die Aufgabe einer breiter angelegten Korpusstudie sein. Im Rahmen dieses Aufsatzes werde ich lediglich auf drei Fragen eingehen:

11 Die Auswertung bezieht sich auf zwei Textausschnitte (je 12000 Wörter) aus autobiographischen Chroniken um 1650: die eine ist von Augustin Güntzer, die andere von Caspar Preis (nähere Textcharakteristika in Volodina 2009, außerdem Hennig 2009). Quelle: Kasseler DFG-Projekt-Korpus „Explizite und elliptische Junktion in der Syntax des Neuhochdeutschen" (Leitung: V. Ágel, M. Hennig).

12 Helene Kottanerin, Denkwürdigkeiten, Wien 1445-1452. Quelle: Text 113: Bonner Korpus http://www.korpora.org/Fnhd/FnhdC.HTML/113.html 
FRAGE 1: Wie frequent sind Nullsubjekt-Strukturen in den untersuchten Texten des FNHD und frühen NHD und welche morphologische Distribution weisen sie auf?

FRAGE 2: Stützt der empirische Befund die These der historischen Kontinuität des Nullsubjekt-Phänomens (vgl. Axel/Weiß 2010)?

FRAGE 3: Gibt es in den untersuchten Sprachstufen auch andere Typen referentieller Nullsubjekte, die im heutigen Deutsch nicht mehr belegt sind, und wodurch ist ihr Vorkommen lizenziert?

ad 1: In dem ausgewerteten FNHD-Text liegt der Anteil covert realisierter Subjektpronomina bei ca. 12\%, der absolute Wert beläuft sich auf 83 Belege. ${ }^{13} \mathrm{Im}$ morphologischen Bereich zeichnet sich eine klare Tendenz bei der Auslassung des Subjektpronomens in der 3.SG und 3.PL ab. In der 3.PL liegt der Anteil von Nullsubjekten sogar deutlich über dem Durchschnittswert. Die gleiche Distribution zeigen auch die Daten aus dem frühen NHD (vgl. Volodina 2009), die Nullsubjektrealisierung in der 3.SG/ $\mathrm{PL}$ ist deutlich prominenter als in den anderen Personen und Numeri. Da die 2.SG im untersuchten FNHD-Text overt nicht realisiert ist, ist auch ein covertes Vorkommen nicht zu erwarten, anders als in der 1.SG und 2.PL, in denen overt realisierte Subjektpronomina sehr häufig sind.

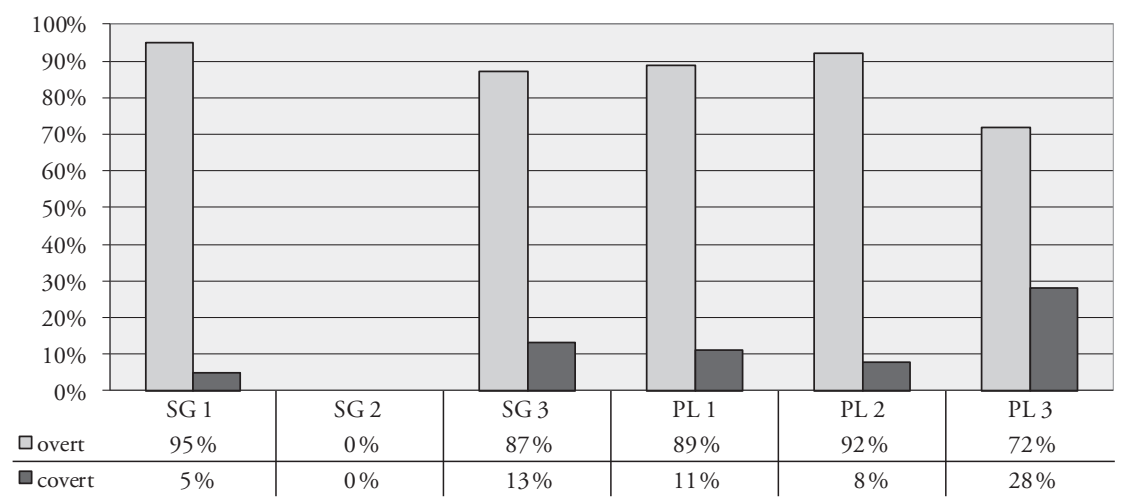

Abb. 1: FNHD-Daten: Person-Numerus-Distribution

In den beiden Texten des frühen NHD dagegen kommen interessanterweise die auch in der heutigen gesprochenen Syntax typischen Auslassungen des Subjektpronomens in der 1.SG und PL im Vorfeld erheblich

13 Koordinationsellipsen haben einen anderen Status als in diesem Aufsatz besprochene Subjektauslassungen, sie sind daher bei dieser Auswertung nicht berücksichtigt worden. 
häufiger vor (vgl. (11a)). Nullsubjekte im Mittelfeld, die als pro zu analysieren sind, können mit den ersteren wegen des niedrigen Vorkommens nicht konkurrieren, sind aber auch noch im frühen NHD mit 6\% (35 Belege) aller Nullsubjekt-Realisierungen (vgl. Volodina 2009: 58) nicht ganz unerheblich (vgl. (11b)):

(11) a. Wir haben auch 12 Schw[e] in in der Mast gehabt. [Ø] Haben ein Schwein davon geschlacht den 25ten Novemberis. (Preis S. 163, zit. nach Volodina 2009: 60)

b. auch ginge $[\varnothing]$ zum tritten fihr deß obern schultzißen hauß (Güntzer, f. 104 v.)

,auch ginge (ich) zum dritten Mal zu dem Haus des oberen Schultheiß‘

ad 2: Wenn die in Axel/Weiß 2010 aufgestellte These nach historischer Kontinuität der Nullsubjekt-Eigenschaft im Deutschen haltbar ist, sollen (im Idealfall) auch die Daten des FNHD die gleiche Tendenz hinsichtlich morphologischer und syntaktischer Muster aufweisen wie im AHD und/ oder in den Dialekten des Deutschen oder theoretisch durch identische Lizenzierungsbedingungen erklärbar sein. Im untersuchten FNHD-Text zeigen sich klare Überschneidungen mit dem AHD (Eggenberger 1961, Axel 2007) nicht nur in morphologischer Hinsicht in Bezug auf das häufige Vorkommen von Nullsubjekten in der 3.SG und PL, sondern auch in syntaktischer Hinsicht: Wie im AHD sind Nullsubjekte im FNHD und frühen NHD fast ausschließlich auf Hauptsätze beschränkt (für einige Ausnahmefälle siehe FRAGE 3). Ein mögliches Problem stellt aber die obligatorische morphologische Beschränkung auf die 2.SG und PL hinsichtlich der Lizenzierung von Nullsubjekten in den heutigen Dialekten dar, was sich gerade komplementär zur Distribution von Nullsubjekten im AHD sowie FNHD und frühen NHD (hier: exemplarisch beschränkt auf die untersuchten Texte) verhält. Ein Faktor ist sicherlich das Fehlen von „flektierten Konjunktionen“ zu dieser Zeit, die die Absenz von pro in Nebensätzen plausibel macht. Das erklärt aber noch nicht vollständig, warum pro in der 2.SG in den untersuchten Texten auch in Hauptsätzen weniger häufig vorkommt als in der 3.SG/PL. Entweder ist das tatsächlich ein Gegenargument - oder Zufall: ${ }^{14}$ Das kann erst eine größere Korpusstudie klären.

14 Für Letzteres sprechen die Ergebnisse einer kleinen Pilotstudie zu Faßnachtsspielen von Hans Sachs, in denen sich die 2.SG tatsächlich wie in den heutigen Dialekten zu verhalten scheint, was die Möglichkeit von pro betrifft. 
ad 3: Schon anhand der ersten Ergebnisse wird deutlich, dass die Vielfalt von Strukturen, in denen Nullsubjekte vorkamen, viel größer ist als heute. Wie an den historischen Daten gezeigt werden kann, kamen im Deutschen immer schon mindestens zwei strukturell unterschiedliche Arten von pronominalen Nullsubjekten vor: Neben zahlreichen satzinitialen Subjektauslassungen (Topik- und Diary-drop-Belege wie in (11a)), die auch für die Syntax der heutigen gesprochenen Sprache typisch sind, sind auch satzinterne Auslassungen von Subjekten nicht unüblich (vgl. (11b)). Die letzteren als pro zu analysierenden Subjektauslassungen sind synchron gesehen z.T. in keiner Varietät des Deutschen mehr grammatisch. Frühere Sprachstufen des Deutschen verfügten über deutlich mehr Arten von Nullsubjekten, deren Erhebung und theoretische Analyse noch aussteht. So finden sich auch in den untersuchten Texten Nullsubjekte, die auf den ersten Blick sowohl gegen die Lizenzierungsbedingungen von Topik-drop im heutigen (gesprochenen) Deutsch als auch gegen die Lizenzierungsbedingungen von pro-drop im AHD und in den heutigen Dialekten verstoßen: (12) a. begerdten an mich ${ }_{j},[\varnothing]_{j}$ sollte mit Machen (Güntzer, f. 60 v.). "sie verlangten von mir, (ich) sollte mit machen."

b. ich solt auf das haws vnd solt versüchen, ob [Ø] ir kran vnd ander ir klainat mocht hinab zu ir bringen (Kottanerin, 13,13).

„ich sollte in das Haus und sollte versuchen, ob (ich) ihre Krone und ihre anderen Kleinodien zu ihr hinab zu bringen vermöchte."

Gegen die Analyse des Nullsubjektpronomens in der 1.SG als pro in (12a) spricht die Realisierung des Nullsubjekts im Vorfeld, gegen die Analyse von Topik-drop die Tatsache, dass der vorliegende V2-Satz kein eigentlicher Hauptsatz ist, sondern in die Struktur des vorherigen eingebettet ist. ${ }^{15}$ Das Nullsubjekt in (12b) kann einerseits kein Topik-drop sein, weil es nicht satzinitial realisiert wird, andererseits auch kein pro, weil pro durch die Flexion in $\mathrm{C}^{0}$ /in der linken Satzklammer lizenziert wird, was in einem Nebensatz mit der Option „flektierte Konjunktion“ einhergehen sollte. Offensichtlich haben sich die Lizenzierungsbedingungen im FNHD und frühen NHD z.T. geändert.

15 Im heutigen Deutsch ist in dieser Position ein overtes Subjektpronomen obligatorisch. 


\section{Fazit und Ausblick}

In den früheren Sprachstufen des Deutschen zeigen sich sowohl eine erstaunliche Konstanz als auch einige bemerkenswerte Veränderungen in Bezug auf die Distribution und die Lizenzierungsbedingungen von referentiellen Nullsubjekten. Diese Fälle umfassen pro-drop, Topik- und Diary-drop, Nullsubjekte in eingebetteten V2-Sätzen und in Nebensätzen, die allerdings weder unter Topik-(bzw. Diary-)Drop noch unter pro-drop subsumiert werden können. Manche dieser Möglichkeiten sind nur für frühere Sprachstufen belegt, andere kommen auch im NHD vor.

Die Entwicklung von Nullsubjekten im Deutschen ist ein spannendes, sowohl empirisch als auch theoretisch vielversprechendes Thema, das einer umfangreichen Korpusuntersuchung bedarf. Die neuen Belege sollen die empirische Belegbasis sowie den theoretischen Horizont von Nullsubjekten in der Diachronie des Deutschen erweitern, u.a. soll die Frage nach dem Nullsubjektstatus des Deutschen geklärt werden.

\section{Literatur}

Axel, Katrin (2007): Studies on Old High German Syntax: Left Sentence Periphery, Verb Placement and Verb-Second. (Linguistik Aktuell/Linguistics Today 112), Amsterdam/Philadelphia.

Axel, Katrin/Weiß, Helmut (2010): What changed where? A plea for the re-evaluation of dialectal evidence, in: Anne Breitbarth et al. (Hg.): Continuity and Change in Grammar, Amsterdam, 13-34.

Bayer, Josef (1984): COMP in Bavarian syntax, in: The Linguistic Review 3, 209-274. Eggenberger, Jakob (1961): Das Subjektspronomen im Althochdeutschen. Ein syntaktischer Beitrag zur Frühgeschichte des deutschen Schrifttums, Chur.

Fleischer, Jürg (2006): Zur Methodologie althochdeutscher Syntaxforschung, in: Beiträge zur Geschichte der deutschen Sprache und Literatur, 128/1: 25-69.

Fleischer, Jürg (2010): Zum Verhältnis von Dialektsyntax und Syntax der Nähekommunikation, in: Vilmos Ágel/Mathilde Hennig (Hg.): Nähe und Distanz im Kontext variationslinguistischer Forschung (Linguistik - Impulse \& Tendenzen 35), Berlin/New York, 85-108.

Fleischer, Jürg/Hinterhölzl, Roland/Solf, Michael (2008): Zum Quellenwert des althochdeutschen Tatian für die Syntaxforschung, in: Zeitschrift für germanistische Linguistik 36, 211-240.

Fries, Norbert (1988): Über das Null-Topik im Deutschen, in: Sprache und Pragmatik $3,19-49$.

Grewendorf, Günther (1989): Ergativity in German, Dordrecht.

Haegeman, Liliane (2000): Adult null subjects in non pro-drop languages, in: MarcAriel Friedemann/Luigi Rizzi (Hg.), The Acquisition of Syntax, Harlow, 129-169. 
Haider, Hubert (2001): Parametrisierung in der Generativen Grammatik, in: Martin Haspelmath et al. (Hg.): Language typology and language universals. An international handbook, (HSK 20.1), Berlin, 283-293.

Held, Karl (1903): Das Verbum ohne pronominales Subjekt in der älteren deutschen Sprache, Nachdruck: New York 1967. Zugl.: Univ. Göttingen, Diss. 1902.

Hennig, Mathilde (2009): Nähe und Distanzierung. Verschriftlichung und Reorganisation des Nähebereichs, Kassel.

Huang, C.-T.J. (1984): On the distribution and reference of empty pronouns, in: Linguistic Inquiry 15, 531-574.

Jaeggli, Osvaldo/Safir, Kenneth J. (1989): The Null Subject Parameter and Parametric Theory, in: Osvaldo Jaeggli/Kenneth J. Safir (Hg.), The Null Subject Parameter, (Studies in Natural. Language and Linguistic Theory 15), Dordrecht.

Kaiser, Georg A./Meisel, Jürgen M. (1991): Subjekte und Null-Subjekte im Französischen, in: Susan Olsen/Gisbert Fanselow (Hg.), DET, COMP und INFL. Zur Syntax funktionaler Kategorien und grammatischer Funktionen, (Linguistische Arbeiten 263), Tübingen, 110-136.

Müller, Gereon (2006): Pro-drop and Impoverishment, in: Patrick Brandt/Eric Fuß (Hg.), Form, Structure and Grammar. A Festschrift Presented to Günther Grewendorf on Occasion of His 60th Birthday, (studia grammatica 63), Berlin, 93-115.

Nübling, Damaris et al. (2006): Historische Sprachwissenschaft des Deutschen. Eine Einführung in die Prinzipien des Sprachwandels, (narr studienbücher), Tübingen.

Rizzi, Luigi (1986): Null Objects in Italian and the theory of pro, in: Linguistic Inquiry $17,501-557$.

Schlachter, Eva (2010): Syntax und Informationsstruktur im Althochdeutschen. Untersuchungen am Beispiel der Isidor-Gruppe. Unveröffentlichte Dissertation, Humboldt-Univestiät Berlin.

Schrodt, Richard (2004): Althochdeutsche Grammatik II: Syntax, Tübingen.

Szczepaniak, Renata (2009): Grammatikalisierung im Deutschen. Eine Einführung, (narr studienbücher), Tübingen.

Volodina, Anna (2009): pro-drop im frühen Neuhochdeutschen, in: Gisela Brandt/ Rainer Hünecke (Hg.), Historische Soziolinguistik des Deutschen IX. Neue Forschungsansätze - Fallstudien - Reflexe konzeptueller Mündlichkeit in Schriftzeugnissen verschiedener soziofunktionaler Gruppen, Stuttgart, 51-66.

Weiß, Helmut (1998): Syntax des Bairischen. Studien zur Grammatik einer natürlichen Sprache, (Linguistische Arbeiten 391), Tübingen.

Weiß, Helmut (2005): Inflected Complementizers in Continental West Germanic Dialects, in: Zeitschrift für Dialektologie und Linguistik, 72, 148-166. 